BIOBUSINESS BRIEFS

TRIAL WATCH

\title{
Tracing investment in drug development for Alzheimer disease
}

The high failure rates of drug $R \& D$ for Alzheimer disease (AD) - and particularly the recent failures of several drugs that target the amyloid cascade in phase III trials - have raised questions about the relative emphasis on particular therapeutic strategies for AD. Here, we present the results of an analysis that aims to inform such debate by mapping $\mathrm{R} \& \mathrm{D}$ investment to the pathophysiological mechanisms of $\mathrm{AD}$, rather than to individual drugs or targets (see Supplementary information S1 (box) for details). The analysis exploits a 'mechanism inventory' for $\mathrm{AD}$, based on a mechanistic model of the disease encoded in biological expression language (Alzheimers Dement. 11, 1329-1339; 2015). This inventory, which has recently been developed (and continues to be enhanced) in the course of the AETIONOMY project (see Further information), includes 126 mechanisms for AD (Bioinformatics btx399, 23 June 2017).

A list of drugs that either underwent or were still undergoing clinical trials for $\mathrm{AD}$ was obtained from the Alzforum database in March 2016 (see Further information). We identified 59 discontinued drugs, 88 drugs in different phases of clinical trials that have not yet been discontinued and 5 approved drugs. Specific information regarding the drug targets of small molecules was extracted using DrugBank in combination with the HGNC and UniProt databases. This enabled each drug to be mapped to the most prominently targeted mechanism in the inventory. All antibodies that have been tested target either the amyloid cascade or tau protein, and were classified accordingly. A substantial proportion (36\%) of drugs could not be mapped to a particular mechanism, and were classified as 'other'.

Investments in the development of these drugs for $\mathrm{AD}$ were estimated based on both the opinions of experts from various companies on clinical trial costs and on publicly available information on completed clinical trials for potential $\mathrm{AD}$ therapies conducted up until March 2016 (Supplementary information S1 (box)). ClinicalTrials.gov was used to gather information about the phase and number of patients enrolled in each trial. We also made a substantial effort to fill in missing information (see Supplementary information S2 (table) for an example). It is likely that many clinical trials have not been registered on ClinicalTrials.gov (particularly earlier in our study period, before registration became standard practice) and so we had to make assumptions about average trial sizes and associated cost per patient for some of the drugs.

Linking the investments in the clinical trials of each drug to their mapped mechanism provides an estimate of the relative amount of investment in each mechanism. FIG. 1 a shows the estimated relative amounts of unsuccessful investment in different mechanisms, based on 37 of the 59 drugs that have been discontinued for which we could identify sufficient information for analysis (see Supplementary information S3 (table)). This analysis indicates that the mechanisms that were the greatest focus of R\&D investment were the amyloid cascade, tau aggregation, neuroinflammation and neurotransmission. FIG. $1 \mathrm{~b}$ shows the focus of current investment in different mechanisms based on 61 of the 88 drugs in ongoing clinical trials for which mechanisms could be assigned (see Supplementary information S4 (table)), indicating relatively little or no investment in some promising novel mechanisms such as targeting endocytosis or autophagy.

The main limitation of our study was the limited access to (or missing) data, which required assumptions to be made

a
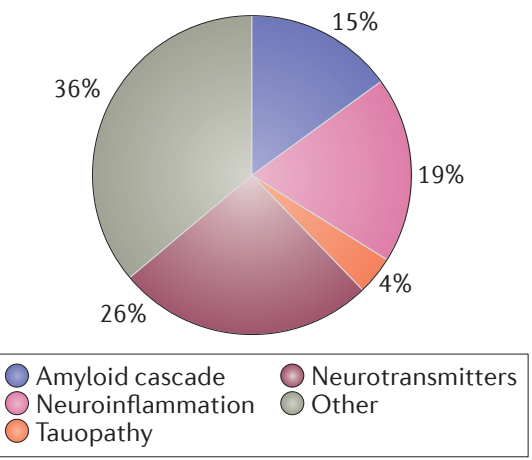

b

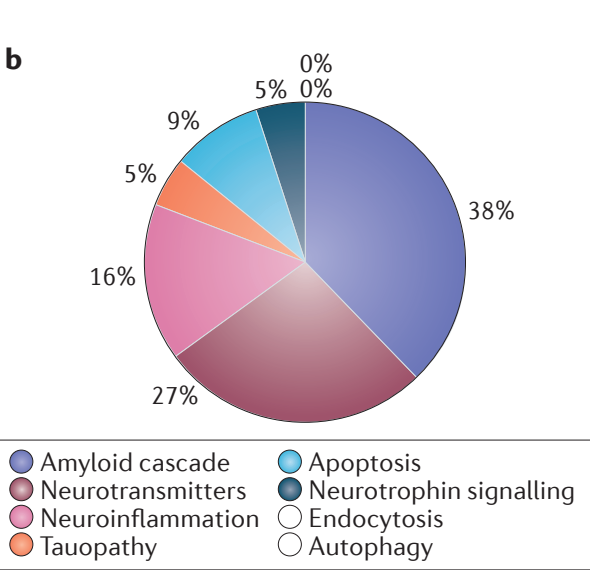

The authors acknowledge support from the Innovative Medicines Initiative grant 115568 (AETIONOMY), and thank R. Karki and C. Ebeling for their support in installation of the MySOL database and queries.

Competing interests statement

The authors declare no competing interests.

FURTHER INFORMATION AETIONOMY project: http://www.aetionomy.eu Alzforum database: http://www.alzforum.org/therapeutics SUPPLEMENTARY INFORMATION See online article: $\underline{\mathrm{S}}$ (box) $\mid \underline{\mathrm{S} 2}$ (table) $\mid \underline{\mathrm{S}} \underline{\underline{N}}$ (table) $\mid \underline{\mathrm{S}}$ (table) ALL LINKS ARE ACTIVE IN THE ONLINE PDF

Figure 1 | Investment in drug development for Alzheimer disease. a | Overview of unsuccessful investment in mechanistic classes based on discontinued drugs. b | Mechanistic focus of investment in ongoing clinical trials. See Supplementary information S1 (box) for details. 